\title{
The Effect of Galega officinalis on Hormonal and Metabolic Profile in a Rat Model of Polycystic Ovary Syndrome
}

\author{
Seyed Hosein Abtahi-Eivari ${ }^{1}$, Maryam Moghimian ${ }^{1}$, Malihe Soltani ${ }^{1}$, Hamed Shoorei ${ }^{2}$, Reza Asghari ${ }^{2}$, \\ Hossein Hajizadeh ${ }^{3}$, Majid Shokoohi ${ }^{2,4^{*}}{ }^{\mathbb{C}}$, Somayye Alami ${ }^{1}$, Faezeh Khalife Ghaderi ${ }^{5}$
}

\begin{abstract}
Objectives: Polycystic ovary syndrome (PCOS) has been considered as one of the most common endocrine diseases among the females in their regenerative age with a prevalence range of 5 to $21 \%$. However, the purpose of the present study was to investigate the protective effect of Galega officinalis on metabolic as well as hormonal parameters in a rodent model of PCOS.

Materials and Methods: Thirty-two Wistar female rats were used (n=8/group) in the study consisting of healthy control and experimental groups. The experimental groups were divided into 3 subgroups, including rats with PCOS which received no treatment (G1), PCOS group in which G. officinalis extract was administered daily at a dose of $200 \mathrm{mg} / \mathrm{kg} /$ orally (G2) for 2 weeks, and PCOS group in which G. officinalis extract was administered daily at a dose of $400 \mathrm{mg} / \mathrm{kg} /$ orally (G3) for 2 weeks. In all experimental groups, a single intramuscular injection of estradiol valerate led to inducing PCOS. After the end of treatment period, rats in all of the studied groups were anesthetized with ketamine/xylazine $(5 / 1 \mathrm{mg} / \mathrm{kg})$, then the blood samples obtained and their serum samples were applied for testing the fasting blood sugar (FBS), insulin, aromatase, and follicle-stimulating hormone (FSH), luteinizing hormone (LH), testosterone, and estrogen. The ovaries of rats were removed and fixed for histopathological examinations.

Results: The serum levels of FBS, insulin, LH, FSH, and testosterone significantly increased in G1 in comparison to healthy rats $(P<0.05)$, while they were all significantly decreased in the treated groups received $G$. officinalis extract compared to rats affected by PCOS $(P<0.05)$. Moreover, the serum level of estrogen and the serum activity of aromatase were both significantly decreased in G1 in comparison to healthy rats $(P<0.05)$, while in treated groups, they were significantly increased compared to G1 which received no treatment $(P<0.05)$. Moreover, the number of follicles in ovaries affected by PCOS decreased, while both concentrations of $G$. officinalis extract could prevent this phenomenon.

Conclusions: It seems that the extract of G. officinalis has a beneficial effect on the levels of the LH, FSH, testosterone, estradiol, aromatase, FBS, and insulin in alleviating the complications of PCOS.

Keywords: Galega Officinalis, PCOS, Steroid hormones, Rat
\end{abstract}

\section{Introduction}

Polycystic ovary syndrome (PCOS) is considered as one of the current disorders of the endocrine system in women of reproductive age (1-3). The epidemiological studies indicated that the incidence of PCOS varies in the world with a range of $2.2 \%$ to $26 \%(2,4)$. Such incidence is $43 \%$ to $46 \%$ in the United States, $37.4 \%$ in Germany, and $16 \%$ in Italy (5). The typical complications of PCOS include polycystic ovaries, elevated luteinizing hormone, hyperandrogenism, chronic anovulation along with oligoamenorrhea, obesity, and infertility (6). PCOS could be taken into account as a metabolism-centered syndrome such as insulin resistance syndrome or some other risk factors, including cardiovascular diseases, hypertension, and/or dyslipidemia (7). Although the mechanism underlying the pathogenesis of PCOS is still elusive, this syndrome is concomitant with hormonal perturbation, such as a diminished concentration of progesterone and heightened levels of testosterone, estrogen and luteinizing hormone (8-10). In patients afflicted with both hyperandrogenism and PCOS, there is a strong association between the hyperinsulinemia and hyperandrogenism as the concentration of insulin shows a strong correlation with adrenal steroid excretion (11). On the other hand, in patients with PCOS, hyperinsulinemia is concurrent with heightened levels of low-density lipoprotein (LDL), very low-density lipoprotein (VLDL), triglyceride, total cholesterol (12) and reduced level of high-density lipoproteins (HDL) (13). Additionally, besides insulin resistance, the metabolism of lipid in females suffered from PCOS may be altered by ovarian- or adrenalexcretion of sex steroid hormones. Effects of sex steroids 
on lipid metabolism are complicated by the activities of both estrogens and androgens which are involved in this scenario $(14,15)$. In addition to metabolic alteration, PCOS is attributed to the alterations occurred in the secretion of reproductive hormones, including the increased ratio of the luteinizing hormone (LH) to the follicle-stimulating hormone (FSH) (i.e. LH/FSH) and circulating androgens contributing to the typical symptoms of PCOS, such as hirsutism, acne, as well as acanthosis nigricans (16).

Herbal medicines have long been used for the treatment of female reproductive disorders as they contain pharmacologically active components showing promising effects in patients with (17) PCOS, diabetes, and cardiovascular diseases $(18,19)$. Galega officinalis is a natural plant belonging to the Middle East (20) and southeastern part of Europe (21). In the Medieval period, it was used as a therapy for diabetes $(20,22)$. One of the main components of $G$. officinalis is alkaloid galegine possessing hypoglycemic properties in animal models (23). Furthermore, it was shown that G. officinalis has a remarkable effect on body weight loss and insulin (24). Therefore, for the treatment of diabetes, the use of this herb can open a new avenue by increasing the sensitivity of tissues to insulin and reducing the tissue damage (19).

However, since PCOS results in damage to organs and tissues of the human body, such as the ovaries, the kernel goal of the study was to examine the effects of $G$. officinalis extract on the damage of ovarian tissue as well as biochemical factors altered as a result of PCOS.

\section{Materials and Methods}

In the present study, 32 adult female Wistar rats (weighing 200-250 g) were used. All rats were procured from Razi Institute, Mashhad, Iran. They were carefully kept in the animal house under standard conditions (temperature $22^{\circ} \mathrm{C}$ and $12 \mathrm{~h} / 12 \mathrm{~h}$ light/dark cycle) and had free access to food and water.

\section{Experimental Groups and Study Design}

Rats were randomly divided into 4 groups ( $n=8$ /group), as one control group and three experimental groups.

1: Healthy or control group (control).

2: PCOS control group (PCO), in which PCOS was induced by an intramuscular injection (IM) of $16 \mathrm{mg} / \mathrm{kg}$ estradiol valerate (Sigma, Germany) (a single dose) which was dissolved in $0.2 \mathrm{ml}$ of sesame oil (25).

3: PCOS group (PG200) was treated with the hydroalcoholic extract of G. officinalis (200 mg/kg/orally/ daily) for 2 weeks.

4: PCOS group (PG400) was treated with the hydroalcoholic extract of G. officinalis (400 mg/kg/orally/ daily) for 2 weeks.

The vaginal smear was examined daily and estrus cycle was confirmed in all rats. After the treatment period, all rats were anesthetized with ketamine/xylazine $(5 / 1 \mathrm{mg} /$ $\mathrm{kg})(19,26)$, then the blood samples were taken from the hearts of all rats and the serum was separated by centrifugation at $3500 \mathrm{~g}$. The obtained sera were kept at $-70^{\circ} \mathrm{C}$ until the further analysis

Preparation of Hydroalcoholic Extract of Galega officinalis To prepare the extract of G. officinalis, half a kilogram of this plant was purchased from Dineh Company (Iran). For extraction, the plant was firstly dissolved in 2 liters of alcohol $50 \%$ and distilled water and then was kept for 48 hours at room temperature on a shaker (Thermo Fisher). Then, the solution was filtered and centrifuged for 5 minutes at $3000 \mathrm{rpm}$. At the end of this process, the resulting solution was poured into an open-top container and the solvent was evaporated. Finally, to achieve an appropriate concentration, the extract was dissolved in normal saline.

\section{Measurement of Serum Glucose Level}

At the commencement of this study, in samples which were obtained from the tip of the vein of rats' tail, the level of fasting blood sugar (FBS) was measured using a portable glucometer. Accordingly, at the end of the experiment period, the serum glucose level was analyzed using a commercial kit (Pars Azmun, Iran). The value was expressed as $\mathrm{mg} / \mathrm{dL}$.

\section{Measurement of Serum Insulin Level}

The measurement of serum insulin concentration was carried out by ELISA (enzyme-linked immunosorbent assay) using a commercial kit specified for rats (Mercodia, Sweden). Serum insulin levels were expressed as $\mathrm{mIU} / \mathrm{mL}$.

Measurement of the Serum Levels of Testosterone, Estrogen, FSH, LH, and Aromatase

Serum hormone levels were quantified using ELISA kits for testosterone and estrogen (Demeditec, Germany). The absorbance was recorded at $405 \mathrm{~nm}$ for analyzing testosterone and estrogen. Correspondingly, the serum levels of aromatase, FSH, as well as LH were also determined using an ELISA kit (Cusabio, China).

Histological Study

The histological examinations were carried out based on previous experimental studies. The ovarian samples were immersed in the formalin $10 \%$ solution for 72 hours. After fixation, they were dehydrated and embedded in paraffin. Then they were cut into 5 microns thick sections to make tissue samples. They were deparaffinized, stained with hematoxylin-eosin (H\&E), then, evaluated the sections of each ovarian tissue $(n=7)$ from the cortex of ovary to the medulla in a spiral and clockwise direction. In each of the ovarian slide, the number of primary Follicles, pre-antral, antral, cystic follicles and yellow body were enumerated. All sections were assessed by an optical microscope $(\mathrm{NIKON})$ at a magnification of $\times 100(27-29)$. 


\section{Statistical Analysis}

The statistical analysis of the present study was carried out using SPSS software 19.0. All data were presented as mean \pm standard error of mean (SEM) and were compared using one-way ANOVA and Tukey post hoc test. $P<0.05$ was considered statistically significant.

\section{Results}

Serum Level of FBS

In PCOS group, the serum level of FBS was significantly higher compared to control group $(P<0.05)$ (Figure 1$)$. In treated groups received 200 and $400 \mathrm{mg} / \mathrm{kg}$ of $\mathrm{G}$. officinalis extract, the serum level of glucose was significantly lower in comparison to the group affected by PCOS $(P<0.05)$.

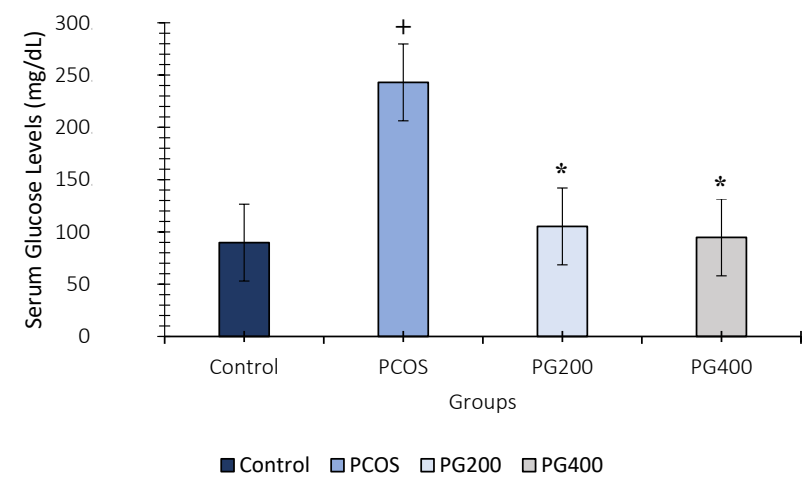

Figure 1. The Serum Level of Glucose in the Experimental and Control Groups.

Control: healthy control group; PCOS: PCOS group that received normal saline by oral gavage; PG200: PCOS group treated with hydroalcoholic extract $(200 \mathrm{mg} / \mathrm{kg})$ of Galega officinalis; PG400: PCOS group treated with hydroalcoholic extract $(400 \mathrm{mg} / \mathrm{kg})$ of Galega officinalis. All data are displayed as mean \pm SE. The asterisk + shows significant difference with control group and the symbol of * means the significant difference with PCOS group $(P<0.05)$.

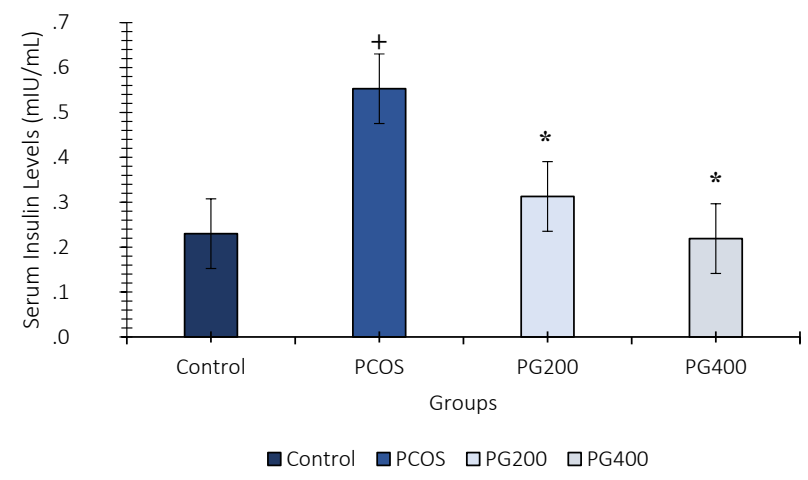

Figure 2. The Serum level of Insulin in the Experimental and Control Groups.

Control: healthy control group; PCOS: PCOS group that received normal saline by oral gavage; PG200: PCOS group treated with hydroalcoholic extract $(200 \mathrm{mg} / \mathrm{kg}$ ) of Galega officinalis; PG400: PCOS group treated with hydroalcoholic extract $(400 \mathrm{mg} / \mathrm{kg})$ of Galega Officinalis. All data are displayed as mean \pm SE. The asterisk + shows significant difference with control group and the symbol of * means the significant difference with PCOS group $(P<0.05)$.
Serum Level of Insulin

In PCOS group, the serum level of insulin was statistically increased in comparison to control group $(P<0.05)$ (Figure 2). However, it was significantly decreased in treated groups, PG 200 and PG 400, in comparison to the group affected by PCOS $(P<0.05)$.

The Serum Levels of Testosterone, Estrogen, LH, FSH and Aromatase

The serum levels of testosterone, estrogen, FSH, and LH were shown in Table 1. According to Table 1, the serum testosterone level was significantly higher in PCOS group compared to control group $(P<0.05)$. Moreover, in both treated groups (PG200 and PG400) the serum levels of testosterone were significantly reduced in comparison to PCOS group $(P<0.05)$. Moreover, there was a significant difference in levels of serum estrogen between PCOS and control groups $(P<0.05)$. Serum estrogen level was increased in treated groups when compared to PCOS group $(P<0.05)$. In PCOS group, there was a significant increase in serum levels of LH and FSH compared to control group $(P<0.05)$. Conversely, in both treated groups, the serum levels of gonadotropin hormones ( $\mathrm{LH}$ and FSH) were significantly decreased in comparison with PCOS group $(P<0.05)$. Likewise, in PCOS group, the serum level of aromatase was significantly decreased compared to control group $(P<0.05)$ (Figure 3$)$. In both groups treated with $G$. officinalis, the serum aromatase level was significantly increased compared to PCOS group $(P<0.05)$.

The Number of Follicles

The number of pre-antral follicles, antral follicles, cystic follicles and yellow bodies is presented in Table 2 and Figure 4 shows the light microscope micrograph of ovaries. However, the number of primary follicles was significantly declined in PCOS group in comparison to control group $(P<0.05)$. Moreover, a significant difference was observed between PCOS group and two treated groups $(P<0.05)$. In PCOS group, the number of pre-antral follicles was significantly declined compared to control group $(P<0.05)$, while the number of pre-antral follicles was significantly higher in both treated groups compared to PCOS group $(P<0.05)$. On the other hand, in PCOS group, enumerating the antral follicles showed a significant decrease in comparison to control group $(P<0.05)$. Furthermore, in both treated groups in comparison to PCOS group, the number of antral follicles significantly increased $(P<0.05)$. On the other hand, in PCOS group, the number of cystic follicles significantly increased when compared to control group $(P<0.05)$. Treatment with $G$. officinalis extract significantly decreased the number of cystic follicles in comparison to rats with PCOS received no therapy $(P<0.05)$. In addition, evaluating the number of yellow bodies showed a significant decrease in PCOS group compared to control group $(P<0.05)$. Additionally, 
Table 1. The Serum Levels of Testosterone, Estrogen, LH, FSH in All of the Studied Groups

\begin{tabular}{|c|c|c|c|c|}
\hline Groups & LH (ng/mL) & FSH (ng/mL) & Testosterone ( $\mathrm{ng} / \mathrm{mL}$ ) & Estrogen $(\mathrm{pg} / \mathrm{mL})$ \\
\hline Control & $1.99 \pm 0.081$ & $2.22 \pm 0.03$ & $0.552 \pm 0.14$ & $52.0 \pm 2.55$ \\
\hline PCOS & $3.26 \pm 0.177^{*}$ & $5.32 \pm 0.50^{*}$ & $3.25 \pm 0.17^{*}$ & $30.25 \pm 1.89^{*}$ \\
\hline PG200 & $2.25 \pm 0.235^{+}$ & $3.25 \pm 0.31^{+}$ & $0.852 \pm 0.035^{+}$ & $38.50 \pm 2.08^{+}$ \\
\hline PG400 & $2.05 \pm 1.70^{+}$ & $2.98 \pm 0.19^{+}$ & $0.670 \pm 0.023^{+}$ & $44.42 \pm 2.57^{+}$ \\
\hline
\end{tabular}

Control: healthy control group; PCOS: PCOS group that received normal saline by oral gavage; PG200: PCOS group treated with hydroalcoholic extract $(200 \mathrm{mg} / \mathrm{kg}$ ) of Galega officinalis, PG400: PCOS group treated with hydroalcoholic extract (400 mg/kg) of G. officinalis. All data are displayed as Mean $\pm \mathrm{SE}$.

The asterisk * shows significant difference with control group and the symbol of + means the significant difference with PCOS group ( $P<0.05)$.

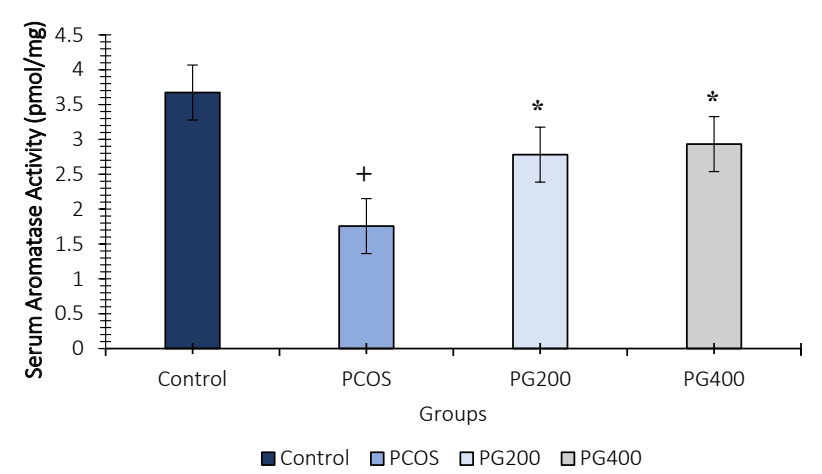

Figure 3. The Serum Activity of Aromatase in the Experimental and Control Groups.

Control: healthy control group; PCOS: PCOS group that received normal saline by oral gavage; PG200: PCOS group treated with hydroalcoholic extract (200 mg/kg) of Galega officinalis; PG400: PCOS group treated with hydroalcoholic extract $(400 \mathrm{mg} / \mathrm{kg})$ of $\mathrm{G}$. officinalis. All data are displayed as mean $\pm \mathrm{SE}$. The asterisk + shows significant difference with control group and the symbol of * means the significant difference with PCOS group $(P<0.05)$.

in both treated groups, PG 200 and PG 400, a significant increase in the number of yellow bodies was observed in comparison to the group affected by PCOS $(P<0.05)$.

\section{Discussion}

Our results showed that G. officinalis had the beneficial effects on the levels of FBS. Similar results were found in a study showed that the decrease in FBS is associated with galegine, a guanidine compound exists in the plant (24). Moreover, Shen et al indicated that G. officinalis has a similar effect to that of metformin because of possessing Bygvandyn composition. Therefore, it would be plausible that G. officinalis could be applied for decreasing the blood sugar (30). Uchiyama et al observed that antioxidants may preclude or postpone the cellular malfunction in diabetes through the protection against toxic levels of glucose (31). Insulin resistance followed by compensatory hyperinsulinemia is considered as the most prominent features among complications resulted from PCOS (32). Insulin resistance has been reported in 25 to $60 \%$ of women afflicted with PCOS (33). It has also been described that G. officinalis is able to decrease hyperinsulinemia (24). Moreover, the serum level of insulin was decreased in the groups treated with $G$. officinalis compared with PCOS group. Such decrease may stem from the existence of complexes such as resin, glycoside, saponins, and steroids. Therefore, antioxidant existing in those compounds can protect the beta cells of the pancreas from damage caused by oxidative stress (19). Some flavonoids can modify the insulin-secreting capacity and modulate beta cell apoptosis. It should be noted that the decrease in antioxidant capacity has been shown to have a correlation with insulin resistance (34).

A number of studies have illustrated that patients with PCOS have higher levels of LH, FSH, and testosterone, while the level of estrogen is lower in patients affected by PCOS (35). It has been indicated that the serum activity of aromatase is declined in PCOS patients (35).

The present study showed that PCOS caused a significant increase in the levels of testosterone, $\mathrm{LH}$, as well as FSH, while the levels of aromatase and estrogen significantly decreased compared to control group. Treatment with $G$. officinalis extract is capable of increasing the serum levels of aromatase and estrogen and decreasing the levels of $\mathrm{LH}, \mathrm{FSH}$, and testosterone. Genazzani et al showed that in

Table 2. The Number of Primary, Per-antral, Antral, Cystic Follicles and Yellow Body in Different Groups

\begin{tabular}{lcccc}
\hline Groups & Primary Follicles & Pre-antral Follicles & Antral Follicles & Cystic Follicles \\
\hline Control & $19.4 \pm 1.14$ & $30 \pm 1.58$ & $17.6 \pm 1.14$ & 0 \\
PCOS & $7.6 \pm 1.14^{*}$ & $7.4 \pm 1.14^{*}$ & $3.8 \pm 0.83^{*}$ & $6.6 \pm 0.54^{*}$ \\
PG200 & $13.4 \pm 1.14^{+}$ & $19 \pm 1.58^{+}$ & $10.4 \pm 1.58^{+}$ & $2.4 \pm 0.54^{+}$ \\
PG400 & $16.8 \pm 0.83^{+}$ & $23 \pm 1.14^{+}$ & $13.6 \pm 1.14^{+}$ & $1.2 \pm 0.83^{+}$ \\
\hline
\end{tabular}

Control: healthy control group; PCOS: PCOS group that received normal saline by oral gavage; PG200: PCOS group treated with hydroalcoholic extract (200 mg/ $\mathrm{kg}$ ) of Galega officinalis; PG400: PCOS group treated with hydroalcoholic extract ( $400 \mathrm{mg} / \mathrm{kg}$ ) of $\mathrm{G}$. officinalis. All data are displayed as mean \pm SE. The asterisk * shows significant difference with control group and the symbol of + means the significant difference with PCOS group $(P<0.05)$. 


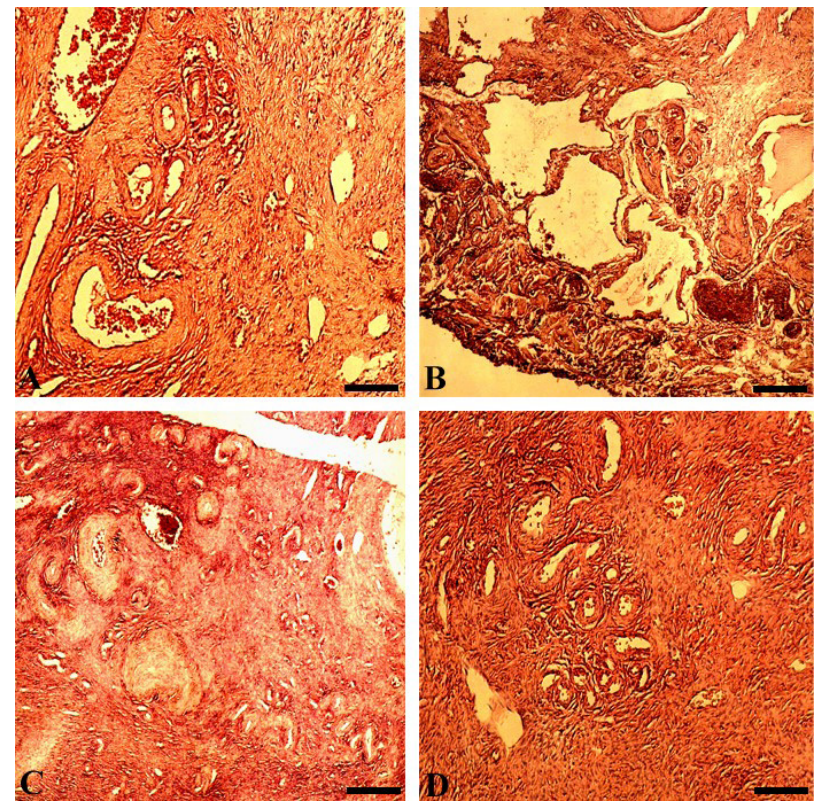

Figure 4. The Histological Findings in Ovaries of the Experimental and the Control Groups.

A: healthy control group; $\mathrm{B}$ : PCOS group that received normal saline by oral gavage; C: PG200: PCOS group treated with hydroalcoholic extract $(200 \mathrm{mg} / \mathrm{kg})$ of Galega officinalis; D: PG400: PCOS group treated with hydroalcoholic extract $(400 \mathrm{mg} / \mathrm{kg})$ of $\mathrm{G}$. officinalis. Scale bar $=100$ micrometers $(x 100)$

patients suffered from PCOS, treatment with metformin can reduce the serum levels of estradiol, LH, as well as insulin (36). On one hand, the reduction in the serum activity of aromatase in PCOS group may be correlated with the decline in serum estrogen level. Christine et al reported that the serum level of estrogen is decreased in PCOS, while the serum levels of LH, FSH, and testosterone are increased in the patients with PCOS (37). Reducing the hyperinsulinemia, whether it is caused by weight loss or the administration of metformin/troglitazone, results in a reduction of plasma levels of $\mathrm{LH}$. However, some studies showed that a decrease in plasma levels of $\mathrm{LH}$ in response to the reduction of insulin may be similar to a phenomenon in which reduced plasma androgen is caused by a reduction in ovarian androgen secretion by insulin (38).

Cytochrome P450c-17a is amongst the enzymes whose activity is increased in some disorders such as PCOS. Furthermore, cytochrome P450c-17a is the main enzyme in the synthesis of androgens, which is directly incited by insulin in patients who suffer from PCOS. Yet, metformin can lead to decrease in the plasma levels of androgens, LH, and IGF-I by reducing the level of insulin, which, in turn, resulted in the reduction of action of this enzyme (39). Insulin is able to prevent the secretion of sex hormonebinding globulin (SHBG) by acting on the liver, hence, the circulating androgens would be increased (40). The incidence of involuntary ovulation can be increased in women with PCOS who take metformin since it can diminish the levels of insulin (41). Mu et al showed that the decline in the plasma concentration of estrogens by troglitazone in PCOS may be as a result of the reduced aromatization which in turn leads to the reduction of circulating insulin level (42). Additionally, Maraca et al reported that in women with PCOS, treatment with metformin could decrease the aromatase activity in response to $\mathrm{FSH}$ (41).

In the present study, in PCOS group, there was a significant decrease in the number of follicles, while the number of cystic follicles was significantly increased. These phenomena might originate from hyperandrogenism that leads to the generation of cystic follicles and reduction of the number of normal follicles (39). Manneras et al reported that PCOS increased the number of atretic antral follicles and decreased the number of healthy antral follicles (43). Moreover, Badawy et al reported that PCOS caused a reduction in the number of healthy follicles in rats with PCOS (44). The results showed that treatment with $G$. officinalis could increase the number of follicles and reduce the number of cystic follicles compared to PCOS group. It can stem from the effects of G. officinalis on serum insulin level and insulin resistance, which are possibly pertained to the presence of Bygvanydyn, flavonoid, saponins in G. officinalis. It has been indicated that these compounds can decrease the insulin resistance and prevent the hyperandrogenism $(24,30)$. However, the effects of G. officinalis on preventing the generation of cystic follicles in the groups treated with PG 200 and PG 400 may be due to anti-hyperandrogenic properties of this herb.

In conclusion, our result showed that $G$. officinalis extract has a striking effect on the level of steroid hormones, the serum aromatase activity, and the serum glucose level in rats. Moreover, the hydroalcoholic extract of G. officinalis could decrease the number of cystic follicles and increase the number of normal follicles. The beneficial effects of $G$. officinalis may be due to the presence of antioxidant and insulin-like agents such as Bygvanydyn, resin, glycoside, and saponins.

\section{Conflict of Interests}

Authors declare that they have no conflict of interests.

\section{Ethical Issues}

All experimental procedures were performed in accordance with Ethics Committee of the Gonabad University of Medical Science (ethics No. IR.GMU. REC.1394.32).

\section{Financial Support}

This study received a grant from Gonabad University of Medical Sciences (GMU), Gonabad, Iran.

\section{Acknowledgments}

Authors are grateful to Gonabad Laboratory Groups. 


\section{References}

1. Jafarisani M, Masoomikarimi M, Kazemi SS, Mirzaeidelaviz S, Naderi Z, Ahmadi R. Effect of Thymus vulgaris Ethanol Extract, on Serum Total Antioxidant in Experimental Induced Poly Cystic Ovarian Syndrome (PCOs) Rats. Int J Health Stud. 2016;2(1):30-34.

2. Tehrani FR, Simbar M, Tohidi M, Hosseinpanah F, Azizi F. The prevalence of polycystic ovary syndrome in a community sample of Iranian population: Iranian PCOS prevalence study. Reprod Biol Endocrinol. 2011;9:39. doi:10.1186/1477-7827-9-39

3. Zheng Q, Li Y, Zhang D, et al. ANP promotes proliferation and inhibits apoptosis of ovarian granulosa cells by NPRA/ PGRMC1/EGFR complex and improves ovary functions of PCOS rats. Cell Death Dis. 2017;8(10):e3145. doi:10.1038/ cddis.2017.494

4. March WA, Moore VM, Willson KJ, Phillips DI, Norman RJ, Davies MJ. The prevalence of polycystic ovary syndrome in a community sample assessed under contrasting diagnostic criteria. Hum Reprod. 2010;25(2):544-551. doi:10.1093/ humrep/dep399

5. Hosseinpanah F, Barzin M, Tehrani FR, Azizi F. The lack of association between polycystic ovary syndrome and metabolic syndrome: Iranian PCOS prevalence study. Clin Endocrinol (Oxf). 2011;75(5):692-697. doi:10.1111/j.13652265.2011.04113.x

6. Anbu AS, Venkatachalam P. Biological macromolecule cross linked TPP-chitosan complex: a novel nanohybrid for improved ovulatory activity against PCOS treatment in female rats. RSC Adv. 2016;6(97):94301-94313. doi:10.1039/ C6RA07228C

7. Hosseinpanah F, Barzin M, Erfani H, Serahati S, Ramezani Tehrani F, Azizi F. Lipid accumulation product and insulin resistance in Iranian PCOS prevalence study. Clin Endocrinol (Oxf). 2014;81(1):52-57. doi:10.1111/cen.12287

8. Holte J, Bergh T, Berne C, Lithell H. Serum lipoprotein lipid profile in women with the polycystic ovary syndrome: relation to anthropometric, endocrine and metabolic variables. Clin Endocrinol (Oxf). 1994;41(4):463-471.

9. Lo JC, Feigenbaum SL, Yang J, Pressman AR, Selby JV, Go AS. Epidemiology and adverse cardiovascular risk profile of diagnosed polycystic ovary syndrome. J Clin Endocrinol Metab. 2006;91(4):1357-1363. doi:10.1210/jc.2005-2430

10. Valkenburg O, Steegers-Theunissen RP, Smedts HP, et al. A more atherogenic serum lipoprotein profile is present in women with polycystic ovary syndrome: a case-control study. J Clin Endocrinol Metab. 2008;93(2):470-476. doi:10.1210/jc.2007-1756

11. Martikainen H, Salmela P, Nuojua-Huttunen S, et al. Adrenal steroidogenesis is related to insulin in hyperandrogenic women. Fertil Steril. 1996;66(4):564-570.

12. Morin-Papunen LC, Koivunen RM, Ruokonen A, Martikainen HK. Metformin therapy improves the menstrual pattern with minimal endocrine and metabolic effects in women with polycystic ovary syndrome. Fertil Steril. 1998;69(4):691-696.

13. Diamanti-Kandarakis E, Mitrakou A, Raptis S, Tolis G, Duleba AJ. The effect of a pure antiandrogen receptor blocker, flutamide, on the lipid profile in the polycystic ovary syndrome. J Clin Endocrinol Metab. 1998;83(8):26992705. doi:10.1210/jcem.83.8.5041

14. Haffner SM, Kushwaha RS, Foster DM, Applebaum-Bowden D, Hazzard WR. Studies on the metabolic mechanism of reduced high density lipoproteins during anabolic steroid therapy. Metabolism. 1983;32(4):413-420.

15. Hazzard WR, Haffner SM, Kushwaha RS, ApplebaumBowden D, Foster DM. Preliminary report: kinetic studies on the modulation of high-density lipoprotein, apolipoprotein, and subfraction metabolism by sex steroids in a postmenopausal woman. Metabolism. 1984;33(9):779784.

16. Pergialiotis V, Konstantopoulos P, Prodromidou A, Florou V, Papantoniou N, Perrea DN. Management of endocrine disease: the impact of subclinical hypothyroidism on anthropometric characteristics, lipid, glucose and hormonal profile of PCOS patients: a systematic review and meta-analysis. Eur J Endocrinol. 2017;176(3):R159-r166. doi:10.1530/eje-16-0611

17. Shoorei H, Khaki A, Ainehchi N, et al. Effects of Matricaria chamomilla Extract on Growth and Maturation of Isolated Mouse Ovarian Follicles in a Three-dimensional Culture System. Chin Med J (Engl). 2018;131(2):218-225. doi:10.4103/0366-6999.222324

18. Arentz S, Abbott JA, Smith CA, Bensoussan A. Herbal medicine for the management of polycystic ovary syndrome (PCOS) and associated oligo/amenorrhoea and hyperandrogenism; a review of the laboratory evidence for effects with corroborative clinical findings. BMC Complement Altern Med. 2014;14:511. doi:10.1186/14726882-14-511

19. Abtahi-Evari SH, Shokoohi M, Abbasi A, Rajabzade A, Shoorei H, Kalarestaghi H. Protective Effect of Galega officinalis Extract on Streptozotocin-Induced Kidney Damage and Biochemical Factor in Diabetic Rats. Crescent Journal of Medical and Biological Sciences. 2017;4(3):108114.

20. Shojaee SS, Vahdati A, Assaei R, Sepehrimanesh M. Effect of Galega officinalis leaf powder and Trigonella foenumgraecum seed powder on blood glucose levels and weight gain in a diabetes mellitus rat model. Comp Clin Path. 2015;24(1):145-148. doi:10.1007/s00580-013-1873-7

21. Rasekh HR, Nazari P, Kamli-Nejad M, Hosseinzadeh L. Acute and subchronic oral toxicity of Galega officinalis in rats. J Ethnopharmacol. 2008;116(1):21-26. doi:10.1016/j. jep.2007.10.030

22. Oubre AY, Carlson TJ, King SR, Reaven GM. From plant to patient: an ethnomedical approach to the identification of new drugs for the treatment of NIDDM. Diabetologia. 1997;40(5):614-617. doi:10.1007/s001250050724

23. Reuter G. [Arginine As The First Stage Of Galegine In Galega officinalis L. On The Biochemistry And Physiology Of Galegine In Galega officinalis L. Iii]. Arch Pharm Ber Dtsch Pharm Ges. 1963;296:516-522.

24. Palit P, Furman BL, Gray AI. Novel weight-reducing activity of Galega officinalis in mice. J Pharm Pharmacol. 1999;51(11):1313-1319.

25. Ouladsahebmadarek E, Khaki A, Khanahmadi S, Ahmadi Ashtiani H, Paknejad P, Ayubi MR. Hormonal and metabolic effects of polyunsaturated fatty acid (omega-3) on polycystic ovary syndrome induced rats under diet. Iran J Basic Med Sci. 2014;17(2):123-127.

26. Roushangar L, Soleymanirad J. Ultrastructural alterations and occurrence of apoptosis in developing follicles exposed to low frequency electromagnetic field in rat ovary. Pak J Biol Sci. 2007;10(24):4413-4419.

27. Roushangar L, Soleymanirad J, Nikpou P, Melli MS. 
Effect of oxytocin injection on folliculogenesis, ovulation and endometrial growth in mice. Iran J Reprod Med. 2009;7(2):91-95.

28. Soltani M, Moghimian M, Abtahi H, Shokoohi M. The Protective Effect of Matricaria chamomilla Extract on Histological Damage and Oxidative Stress Induced by Torsion/Detorsion in Adult Rat Ovary. Int J Womens Health Reprod Sci. 2017;5(3):187-192. doi:10.15296/ijwhr.2017.34

29. Roushangar L, Soleymanirad J, Afsordeh K. Maternal tamoxifen treatment alters oocyte differentiation in the neonatal mice: inhibition of oocyte development and decreased folliculogenesis. J Obstet Gynaecol Res. 2010;36(2):224-231. doi:10.1111/j.1447-0756.2009.01129.x

30. Shen M. A second look at the ancient drug: new insights into metformin. Ann Transl Med. 2014;2(6):51. doi:10.3978/j. issn.2305-5839.2014.06.14

31. Uchiyama K, Naito Y, Hasegawa G, Nakamura N, Takahashi J, Yoshikawa T. Astaxanthin protects beta-cells against glucose toxicity in diabetic $\mathrm{db} / \mathrm{db}$ mice. Redox Rep. 2002;7(5):290-293. doi:10.1179/135100002125000811

32. Kolodziejczyk B, Duleba AJ, Spaczynski RZ, Pawelczyk L. Metformin therapy decreases hyperandrogenism and hyperinsulinemia in women with polycystic ovary syndrome. Fertil Steril. 2000;73(6):1149-1154.

33. Yeon Lee J, Baw CK, Gupta S, Aziz N, Agarwal A. Role of oxidative stress in polycystic ovary syndrome. Curr Womens Health Rev. 2010;6(2):96-107. doi:10.2174/157340410791321336

34. Pinent M, Castell A, Baiges I, Montagut G, Arola L, Ardevol A. Bioactivity of Flavonoids on Insulin-Secreting Cells. Compr Rev Food Sci Food Saf. 2008;7(4):299-308. doi:10.1111/j.1541-4337.2008.00048.x

35. Patel K, Coffler MS, Dahan MH, Malcom PJ, Deutsch R, Chang RJ. Relationship of GnRH-stimulated LH release to episodic LH secretion and baseline endocrine-metabolic measures in women with polycystic ovary syndrome. Clin Endocrinol (Oxf). 2004;60(1):67-74.

36. Genazzani AD, Lanzoni C, Ricchieri F, Baraldi E, Casarosa E, Jasonni VM. Metformin administration is more effective when non-obese patients with polycystic ovary syndrome show both hyperandrogenism and hyperinsulinemia. Gynecol Endocrinol. 2007;23(3):146-
152. doi:10.1080/09513590701214398

37. Cook CL, Siow Y, Brenner AG, Fallat ME. Relationship between serum mullerian-inhibiting substance and other reproductive hormones in untreated women with polycystic ovary syndrome and normal women. Fertil Steril. 2002;77(1):141-146.

38. Pastor CL, Griffin-Korf ML, Aloi JA, Evans WS, Marshall JC. Polycystic ovary syndrome: evidence for reduced sensitivity of the gonadotropin-releasing hormone pulse generator to inhibition by estradiol and progesterone. J Clin Endocrinol Metab. 1998;83(2):582-590. doi:10.1210/ jcem.83.2.4604

39. De Leo V, la Marca A, Ditto A, Morgante G, Cianci A. Effects of metformin on gonadotropin-induced ovulation in women with polycystic ovary syndrome. Fertil Steril. 1999;72(2):282-285.

40. Nestler JE, Powers LP, Matt DW, et al. A direct effect of hyperinsulinemia on serum sex hormone-binding globulin levels in obese women with the polycystic ovary syndrome. J Clin Endocrinol Metab. 1991;72(1):83-89. doi:10.1210/ jcem-72-1-83

41. la Marca A, Morgante G, Palumbo M, Cianci A, Petraglia F, De Leo V. Insulin-lowering treatment reduces aromatase activity in response to follicle-stimulating hormone in women with polycystic ovary syndrome. Fertil Steril. 2002;78(6):1234-1239.

42. $\mathrm{Mu} \mathrm{YM}$, Yanase $\mathrm{T}$, Nishi $\mathrm{Y}$, et al. Insulin sensitizer, troglitazone, directly inhibits aromatase activity in human ovarian granulosa cells. Biochem Biophys Res Commun. 2000;271(3):710-713. doi:10.1006/bbrc.2000.2701

43. Manneras L, Cajander S, Lonn M, Stener-Victorin E. Acupuncture and exercise restore adipose tissue expression of sympathetic markers and improve ovarian morphology in rats with dihydrotestosterone-induced PCOS. Am J Physiol Regul Integr Comp Physiol. 2009;296(4):R11241131. doi:10.1152/ajpregu.90947.2008

44. Badawy A, Abdel Aal I, Abulatta M. Clomiphene citrate or letrozole for ovulation induction in women with polycystic ovarian syndrome: a prospective randomized trial. Fertil Steril. 2009;92(3):849-852. doi:10.1016/j. fertnstert.2007.02.062

(c) 2018 The Author (s); This is an open-access article distributed under the terms of the Creative Commons Attribution License (http://creativecommons.org/licenses/by/4.0), which permits unrestricted use, distribution, and reproduction in any medium, provided the original work is properly cited. 